\title{
The Prognostic Value of Cell-Free DNA in Advanced Non-Small-Cell Lung Cancer
}

\author{
Anneli Dowler Nygaard ${ }^{1 *}$, Karen-Lise Garm Spindler ${ }^{1}$, Niels Pallisgaard ${ }^{2}$, Rikke Fredslund Andersen², \\ Anders Jakobsen ${ }^{1}$
}

${ }^{1}$ Department of Oncology, Vejle Hospital, Vejle, Denmark; ${ }^{2}$ Department of Biochemistry, Vejle Hospital, Vejle, Denmark. Email: *anneli.nygaard@rsyd.dk

Received June $21^{\text {st }}, 2013$; revised July $23^{\text {rd }}, 2013$; accepted July $30^{\text {th }}, 2013$

Copyright (C) 2013 Anneli Dowler Nygaard et al. This is an open access article distributed under the Creative Commons Attribution License, which permits unrestricted use, distribution, and reproduction in any medium, provided the original work is properly cited.

\begin{abstract}
Background: Cell-free DNA (cfDNA) holds promise as a tumor marker of clinical importance. We aimed to investigate the prognostic value of baseline cfDNA in non small-cell lung cancer (NSCLC). Material and Methods: During a three-year period, patients with newly diagnosed, previously untreated advanced NSCLC were included in a consecutive, prospective marker-trial. Plasma was isolated from a pre-treatment peripheral blood sample and the level of total cfDNA was measured by an in-house assay qPCR-method. The treatment comprised carboplatin (AUC 5) intravenously day 1$)$, and vinorelbine $\left(30 \mathrm{mg} / \mathrm{m}^{2}\right.$ intravenously day 1 and $60 \mathrm{mg} / \mathrm{m}^{2}$ perorally day 8$) \mathrm{q} 3 \mathrm{w}$ for a maximum of six cycles. The primary end-point was overall survival (OS). Secondary end-points were progression free survival (PFS) and overall response rate (ORR). Results: 245 patients were included and received a minimum of 1 cycle of chemotherapy (median 4). The median OS was 8.9 months, the median PFS by intention to treat 5.4 months and the ORR was $25 \%$. The patients were divided into four groups based on quartiles of cfDNA and subsequently dichotomized by the 75th percentile revealing a significantly worse prognosis for patients in the upper 75th percentile (median OS 4.9 months) compared to patients with lower levels (10.0 months) (HR 2.1, 95\%CI $1.4-3.1, p<0.0001)$. A multivariate analysis confirmed the independent prognostic value of cfDNA. A subgroup analysis of patients with high cfDNA and poor performance status $(\mathrm{PS}=2)$ identified a group of patients with even worse prognosis (median OS 2.0 versus 9.1 months, HR 3.6, 95\%CI $1.4-9.2, p<0.0001$ ). Similar and significant results were found when comparing level of cfDNA and PFS. Conclusions: High pre-treatment level of cfDNA seems to have a strong prognostic impact in patients with newly diagnosed advanced NSCLC. Combined with PS it identifies a patient group with minimal or no benefit of chemotherapy.
\end{abstract}

Keywords: Cell-Free DNA; Prognostic; NSCLC; Circulating DNA

\section{Introduction}

Non-small-cell lung cancer (NSCLC) is one of the most common malignant diseases worldwide, and one of the leading causes of cancer related deaths [1]. When diagnosed at a localized stage, curatively intended treatment by surgery may be an option, but most patients have advanced disease at the time of diagnosis, where the only treatment available is palliative systemic treatment [2]. The effect, however, is moderate with only a minor subgroup benefiting from the treatment, and improving the patient selection is crucial. Consequently, predictive and prognostic tumor markers are gaining mounting importance and interest, but so far only few reliable markers

"Corresponding author. have been identified and used in clinical settings.

DNA detectable in plasma and serum, referred to as cell free DNA (cfDNA) is a potential marker which was discovered in 1948 by Mandel and Metais. It gained little interest until the 1960's where high levels of cfDNA were demonstrated in patients with systemic lupus erythematosus [3]. The first evidence of increased levels of cfDNA in cancer patients was published in the seventies $[3,4]$. Since then, cfDNA in cancer patients has been investigated in several settings, but so far it has not been clinically implemented. The biological mechanisms behind the presence of cfDNA in blood are not yet fully understood. It is present under both normal and pathological conditions, probably due to apoptosis and necrosis leading to release into the bloodstream, but also active 
release from the cells has been suggested. Under malignnant conditions, the level of cfDNA is increased, possibly due to higher activity in the aforementioned processes, but additional hypotheses regarding increased release of DNA from tumor-surrounding tissue due to tumor invasion have also been suggested [5-7]. Furthermore, it has been proposed that some of the cfDNA may origin from circulating tumor cells (CTC:s) lysing in the circulation [5].

The fact that the level increase of cfDNA during malignant conditions makes it feasible as a marker of disease activity and prognosis. The baseline level of cfDNA as well as variations during the course of treatment and disease may provide important information regarding the behavior of the disease, and the prognosis.

But despite numerous studies and different approaches, no consensus upon the role of cfDNA has been reached. Although there is agreement of its potential as a biomarker, the question of whether it is diagnostic, predictive, or prognostic remains unanswered and requires further investigation [8].

Further studies in larger, homogeneous cohorts under standardized procedures and techniques are needed in order to determine the clinical role of cfDNA.

In the present study, we aimed to address the prognostic value of baseline plasma cfDNA, in a large cohort of patients with advanced or disseminated NSCLC, treated with first line chemotherapy.

\section{Material and Methods}

\subsection{Patients}

During a three-year period (2007-2010) patients with newly diagnosed advanced NSCLC were prospectively included in a marker-trial at the Department of Oncology, Vejle Hospital. The inclusion criteria were; histopathologically verified NSCLC, stage III-IV disease, no previous chemotherapy, and performance status (PS) allowing chemotherapy (PS $\leq 2)$. The patients were treated with first-line chemotherapy according to standard guidelines of our department and comprised carboplatin (AUC $5)$, administered intravenously on day 1 every three weeks) in combination with vinorelbine $\left(30 \mathrm{mg} / \mathrm{m}^{2}\right.$ administered intravenously on day 1 and $60 \mathrm{mg} / \mathrm{m}^{2}$ perorally on day 8 every three weeks) for a maximum of six cycles. Palliative radiotherapy was given when indicated. In a few cases $(n=14)$ the initial chemotherapy was followed by curatively intended radiotherapy due to significant reduction in disease burden. A pre-treatment blood sample was collected within two weeks ahead of treatment start and subsequently analyzed for the baseline level of cfDNA. All patients had a baseline CT of the chest and upper abdomen within a month before the first treatment.
The primary endpoint was overall survival (OS). Secondary endpoints were progression free survival (PFS) and response rate (RR). Objective response was evaluated according to RECIST v. 1.0 by CT scans of the chest and upper abdomen after every two cycles and registered as best overall response [9]. Patients who had partial (PR) or complete response (CR) were characterized as responders. Disease control (DC) was defined as response or stable disease (SD). Written informed consent was obtained before enrolment and the study was conducted in accordance with Danish law after approval by the Regional Scientific Ethical Committee for Southern Denmark.

\subsection{Analyses of cfDNA in Plasma}

From the pre-treatment peripheral blood sample which was collected in EDTA-tubes, plasma was obtained by centrifugation at $2000 \times \mathrm{g}$ for 10 minutes and stored at $-80^{\circ} \mathrm{C}$. The plasma was separated within 2 hours after sampling. Total nucleic acid was obtained from $1.0 \mathrm{ml}$ plasma by the use of a QIAsymphony virus/bacteria midi-kit on a QIAsymphony robot (Qiagen) according to the manufacturer's instructions. To determine the level of cfDNA, the amount of the peptidylprolyl isomerase Agene (PPIA), was measured by qPCR using an in-house assay providing plasma-sample DNA, which subsequently was converted to number of DNA alleles $/ \mathrm{ml}$. The method has been described in detail previously [10].

\subsection{Statistics}

The association between objective response rates and the level of cfDNA was investigated by Wilcoxon Rank-sum or $\mathrm{X}^{2}$-test. The same methods were used for associations between patient characteristics and cfDNA. Survival analyses were performed by the Kaplan-Meier method and differences according to level of cfDNA were estimated by the log-rank-test. Hazard ratios were evaluated by the Mantel-Haenszel-method.

OS was defined as the time from date of first treatment until date of death by any cause. PFS was defined as the time from date of first treatment until date of progression or death by any cause. Patients who had neither progressed nor died were censored. Date of last observation was the 1st of November 2012.

The independent values of different variables were investigated in multivariate Cox regression analyses. Known prognostic variables and variables with a $p$-value $<0.2$ in univariate analyses were included in the model.

Two-sided $p$-values $<0.05$ were considered significant. Statistics were performed with NCSS statistical software (NCSS Statistical Software, Kaysville, UT, USA, version 07.1.15, 2009). 


\section{Results}

\subsection{Pre-Treatment Characteristics and Bio-Marker Levels}

Patient characteristics are demonstrated in Table 1. A total of 245 patients were included in the analyses. All patients had advanced disease at the time of diagnosis and were candidates for palliative treatment.

The median level of cfDNA was 3212 alleles $/ \mathrm{ml}$ (range 478-1048538). The correlation between patient characteristics at baseline and the level of cfDNA was

Table 1. Patient characteristics.

\begin{tabular}{|c|c|c|}
\hline & $\begin{array}{c}\text { Number } \\
(n=245)\end{array}$ & $\begin{array}{c}\text { Percent } \\
(100)\end{array}$ \\
\hline \multicolumn{3}{|l|}{ Age } \\
\hline Median (range) & \multicolumn{2}{|c|}{$66(40-80)$} \\
\hline \multicolumn{3}{|l|}{ Gender } \\
\hline Male & 151 & 62 \\
\hline Female & 94 & 38 \\
\hline \multicolumn{3}{|l|}{ Histology } \\
\hline Adenocarcinomas & 149 & 61 \\
\hline Squamous cell carcinoma & 75 & 31 \\
\hline Large cell carcinoma & 8 & 3 \\
\hline Other & 13 & 5 \\
\hline \multicolumn{3}{|l|}{ Stage } \\
\hline III & 57 & 23 \\
\hline IV & 188 & 77 \\
\hline \multicolumn{3}{|l|}{ Metastatic sites } \\
\hline 0 & 115 & 47 \\
\hline $1-2$ & 118 & 48 \\
\hline$>2$ & 12 & 5 \\
\hline \multicolumn{3}{|c|}{$\mathrm{ECOG}^{*}$ performance status at baseline } \\
\hline 0 & 88 & 36 \\
\hline 1 & 125 & 51 \\
\hline 2 & 32 & 13 \\
\hline \multicolumn{3}{|l|}{ LDH at baseline $(\mathrm{n}=230)$} \\
\hline Normal & 140 & 61 \\
\hline Elevated & 90 & 39 \\
\hline \multicolumn{3}{|l|}{ Number of cycles } \\
\hline Median (range) & \multicolumn{2}{|c|}{$4(1-6)$} \\
\hline \multicolumn{3}{|l|}{ Radiotherapy } \\
\hline None & 192 & 78 \\
\hline Palliative & 39 & 16 \\
\hline Curative & 14 & 6 \\
\hline
\end{tabular}

${ }^{*}$ ECOG $=$ Eastern Cooperative Oncology Group. investigated and revealed a significantly higher $(p=$ $0.015)$ median level of cfDNA among patients in PS 2 (4152 alleles/ml) than PS 0 - 1 (3115 alleles/ml). Furthermore, patients with increased LDH at baseline had a significantly higher $(p<0.0001)$ median level of cfDNA (2681 alleles/ml) than patients with normal LDH (4370 alleles $/ \mathrm{ml})$.

\subsection{Survival Analyses}

The median OS for the entire group was 8.9 months (95\%CI 7.0 - 9.7 months). Ten patients were still alive at the end of the observation period and censored. The median PFS was 5.4 months $(95 \%$ CI 4.8 - 5.6) with five patients censored. The patients were divided into four groups by the level of cfDNA. Cut-off was based on quartiles (lower 1st quartile at 1910 alleles $/ \mathrm{ml}$, median 3212 alleles $/ \mathrm{ml}$ and upper 4th quartile at 6062 alleles $/ \mathrm{ml}$ ). A clear association between high levels of cfDNA and poor survival was demonstrated (Figure 1). When studying the survival curves, it was obvious that the patients in the upper quartile had the worse prognosis. The median OS of this group was only 4.9 months, whereas the median OS in the lower three quartiles ranged from 8.0 11.3 months, leading us to further investigation by dichotomizing OS by the upper 75th percentile. The Kaplan-Meier curves showed a significantly shorter OS (HR: $2.1,95 \%$ CI $1.4-3.0, p<0.0001)$ of patients in the upper 75th percentile (median 4.9, 95\%CI 3.7 - 6.8 months) than patients with a cfDNA-level below the upper 75th percentile (median 10.0, 95\% CI 8.9 - 11.4 months).

A similar correlation between levels of cfDNA and PFS was seen when patients were grouped by both quartiles $(p=0.0005)$ and dichotomized by the upper 75 th percentile $(p<0.0001)$. Survival curves and data are demonstrated in Figure 1.

\subsection{Multivariate Analyses}

To examine the independent effect of the cfDNA-level, multivariate analyses of both OS and PFS were performed. The model was defined by including known prognostic markers and potentially prognostic patient characteristics with a $p$-value below 0.2 when tested in univariate analyses of OS and PFS. The final model is demonstrated in Table 2. Elevated LDH and adenocarcinomas were independently correlated with a shorter OS, whereas neither stage nor the presence of distant metastases showed any significant correlation with outcome. cfDNA and PS remained independent prognostic factors of both OS and PFS.

\subsection{Subgroup Analyses}

The correlation between high levels of cfDNA, impaired prognosis, and poor performance status inspired to a 


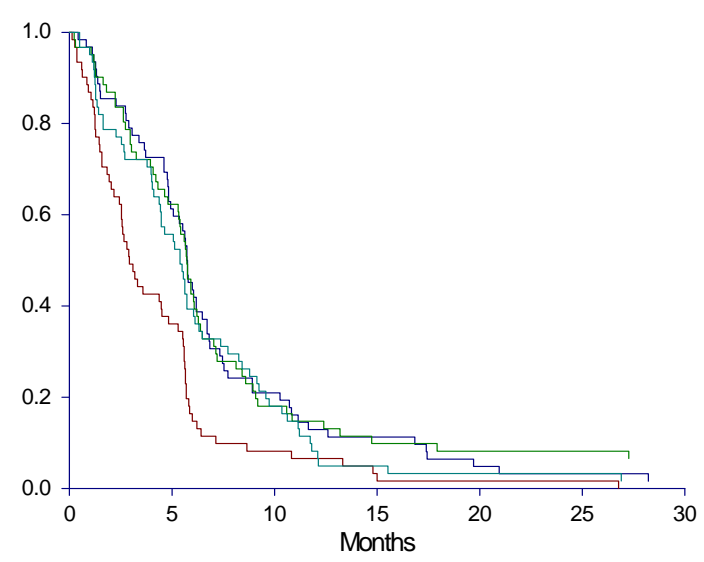

(a)

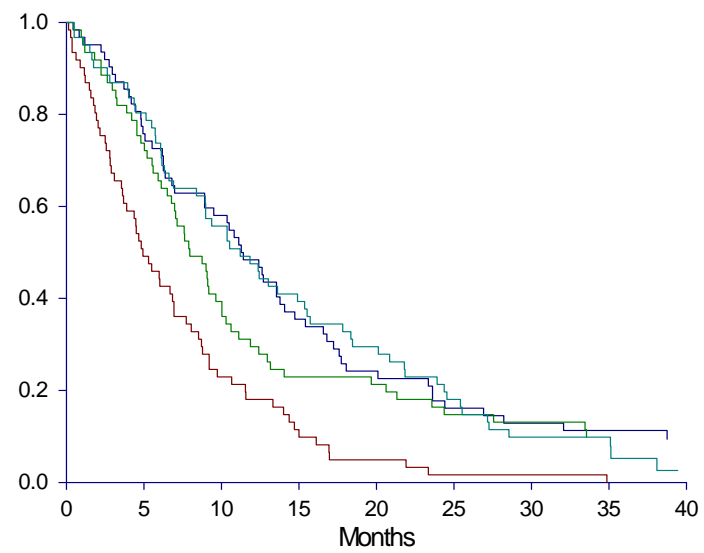

(c)

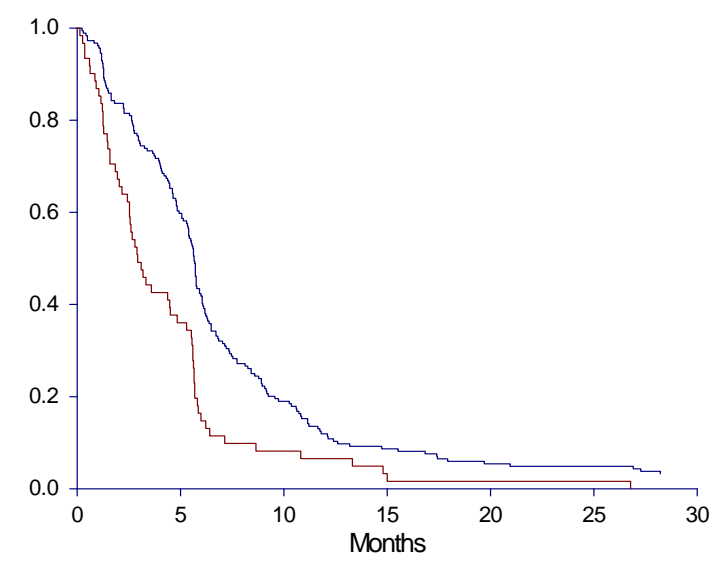

(b)

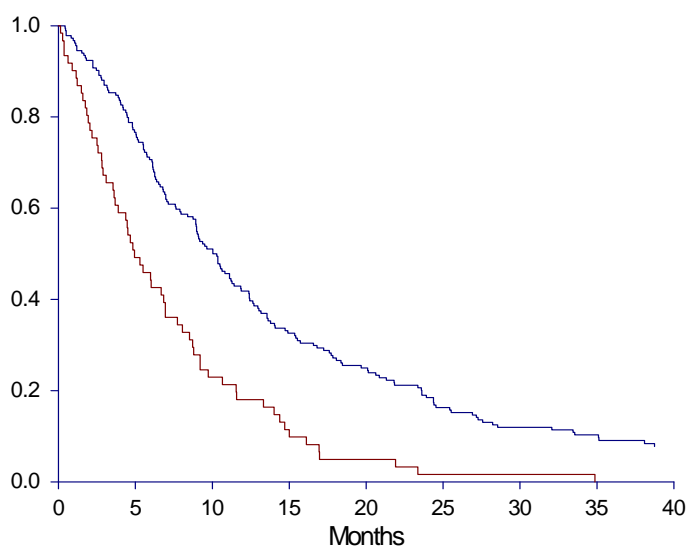

(d)

Figure 1. Progression free survival (PFS) and overall survival (OS) in months, according to level of baseline cfDNA. A: PFS according to quartiles of cfDNA. Median PFS < 25\%: 5.4 (4.4 - 5.7), 25\% - 50\%: 5.7 (4.9 - 6.2), 50\% - $75 \%$ : 5.7 (5.3 - 6.1), $>75 \%$ : 2.9 (2.5 - 4.5), $p=0.0005$. B: PFS according to 75th percentile of cfDNA. Median PFS < 75\%: 5.7 (5.3 - 5.8$)$, >75\%: 2.9 (2.5 - 4.5), HR: 1.80 (1.27 - 2.54), $p<0.0001$. C: OS according to quartiles of cfDNA. Median OS <25\%: $11.2(8.9-14.9), 25 \%$ - $50 \%$ : $11.3(8.9$ - 13.8), 50\% - 75\%: $8.0(6.8$ - 9.6), >75\%: 4.9 (3.7 - 6.8), $p<0.0001$. D: OS according to 75 th percentile of cfDNA. Median OS < 75\%: 10.0 (8.9 - 11.4), >75\%: 4.9 (3.7 - 6.8), HR: 2.08 (1.44 - 2.99), p < 0.0001. A + C: Blue line < 25\%, dark green line $25 \%-50 \%$, light green line $50 \%-75 \%$, red line $>75 \%$. B + D: Blue line = cfDNA below the 75 th percentile; Red line $=$ cfDNA above the 75 th percentile.

subgroup analysis of patients categorized by cfDNA above the 75 th percentile combined with PS $=2$ at baseline. These criteria were met by 15 patients. As demonstrated in Figure 2 both OS and PFS were investigated, revealing a remarkably worse prognosis for the subgroup compared to the other patients. In the subgroup the median OS was only 2.0 months $(95 \%$ CI 0.9 - 2.6) compared to 9.1 months $(95 \% \mathrm{CI} 7.6-10.3)$ in the other group (HR: 3.6, 95\%CI $1.4-9.2, p<0.0001$ ). The PFS was also significantly worse in the subgroup with a median PFS of $1.5(95 \% \mathrm{CI} 0.9$ - 2.0) versus 5.6 months $(95 \%$ CI $5.1-5.7)$ in the other group (HR: $4.0,95 \%$ CI 1.5 $-10.7, p<0.0001)$.

\subsection{Response to Chemotherapy}

All patients available for objective response evaluation were included in the response analyses. Four patients did not fulfill evaluation criteria and were excluded. The overall response rate (ORR) was $25 \%$. When considering response (CR + PR versus $\mathrm{SD}$ and progression (PD)) according to level of cfDNA, no significant relation was found (data not shown).

\section{Discussion}

The intriguing possibility of extracting tumor DNA from a simple blood sample has raised grand expectations of clinical application. The perspective of a "liquid biopsy" is prepossessing, especially in lung cancer where access to tumor material by conventional methods often proves difficult. So far, tumor tissue has been the primary source of tumor DNA and thus material for genetic analyses. This approach has its advantages, but it requires a representative sample and is often associated with significant inconvenience to the patient. Beyond the patient friendly 
Table 2. Multivariate cox regression analyses of progression free survival (PFS) and overall survival (OS).

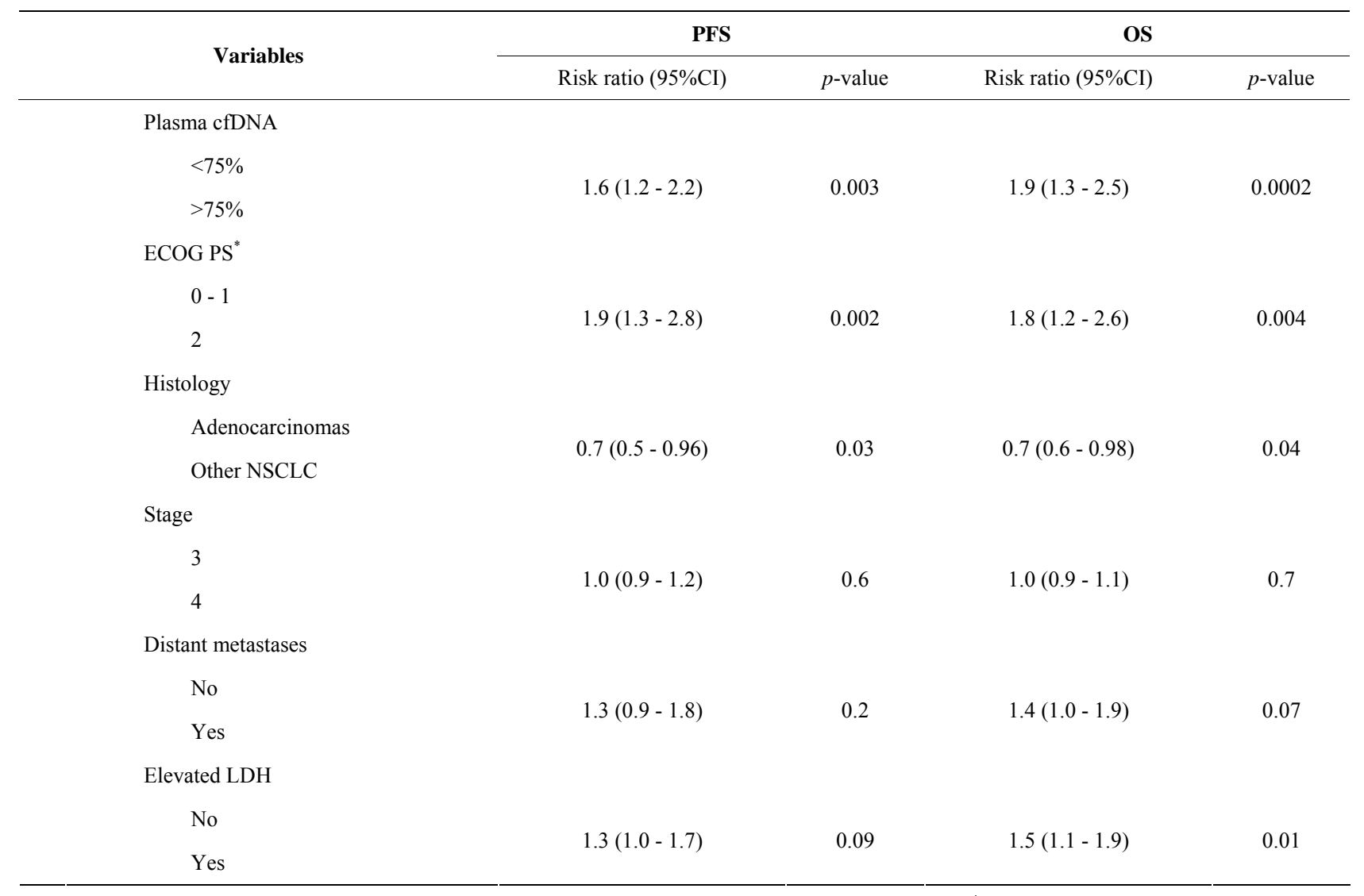

All variables were dichotomized. The Risk Ratio refers to moving from the reference group to the other group; ${ }^{*}$ ECOG PS = Eastern Cooperative Oncology Group Performance Status.

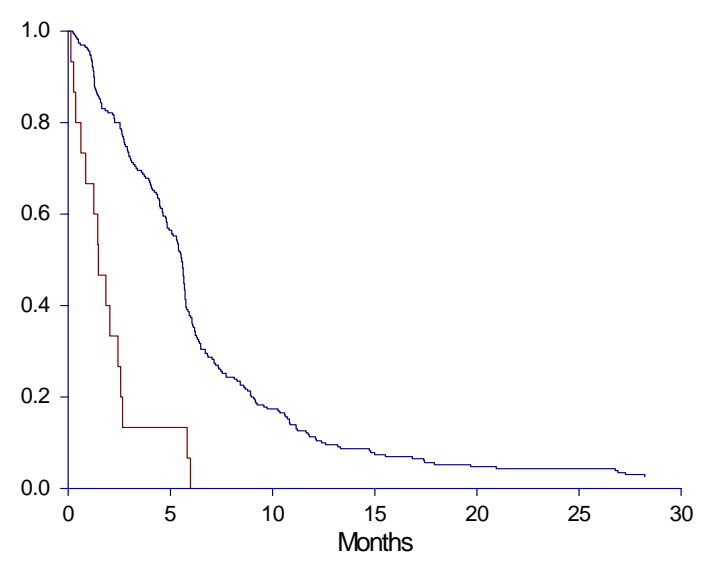

(a)

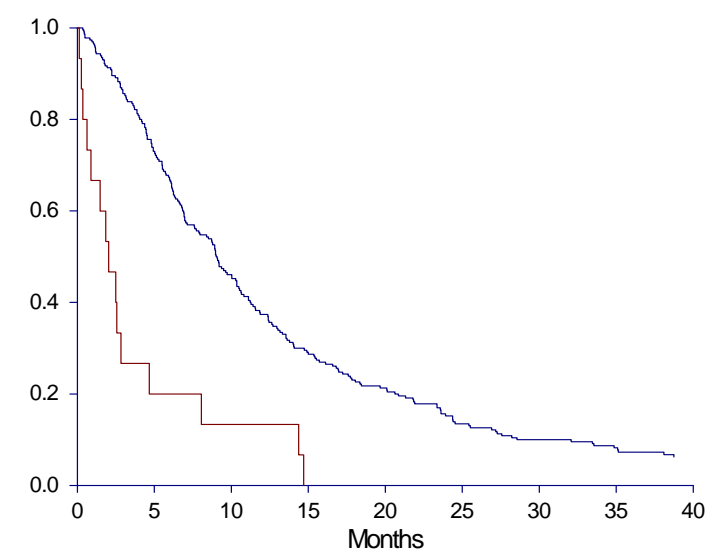

(b)

Figure 2. Progression free survival (PFS) and overall survival (OS) in months, in the subgroup-analysis. A: PFS in high-risk subgroup versus all other patients. Median PFS High risk group: 1.5 (0.9 - 2.0), All other patients: 5.6 (5.1 - 5.7), HR: 3.98 (1.47 - 10.74), $p$ < 0.0001. B: OS in high-risk subgroup versus all other patients. Median OS High risk group: 2.0 (0.9 - 2.6), All other patients: 9.1 (7.6 - 10.3), HR: $3.58(1.39-9.22), p<0.0001$. Patients with cfDNA above the 75th percentile and PS $=2$ were identified as a potential high risk group, and survival analyses of this group $(n=15)$ versus all other patients $(n=231)$ were performed. Red line $=$ High risk group; Blue line $=$ All other patients.

and relatively uncomplicated procedure of blood sampling, blood samples also allow repetitive measurements and thus closely genetic monitoring of the tumor, an im- portant aspect in this era of targeted therapy. These considerations inspired the present study with the main objective of investigating the prognostic value of cfDNA in 
plasma.

To measure the level of cfDNA, an in-house assay qPCR-method targeting the PPIA-gene was used. The method has been validated and used in comparable settings in the study of cfDNA in metastatic colorectal cancer [10]. The target gene (PPIA) is tumor-independent and present in both tumor-and normal tissue, thus providing a measure of the total level of cfDNA present in the blood stream. This aspect has been the subject of many discussions regarding the most appropriate way of determining the level of cfDNA. Since the method provides a non-tumor specific estimate of cfDNA, it has been argued that this approach is insufficient in order to foresee tumor related events such as progression and death. Previous studies have used different methods and target genes which to some extent may explain the diverging results [11]. One of the most common target genes is the human telomerase reverse transcriptase (hTERT), which is known to be over-expressed in most, but not all, NSCLC tumors [12]. Camps et al. managed to demonstrate a correlation between increased hTERT in serum and poor prognosis [13]. Similar results in plasma have also been reported, but the results have not been validated by other authors $[14,15]$. In this cohort of patients with known advanced lung cancer, we find the use of PPIA feasible, as it does not depend on tumor-related genes and therefore provides an estimate of the total cfDNA [16].

The clinical role of cfDNA in lung cancer remains to be defined. In addition to the aforementioned methodological discrepancies, there are several problems in the comparison of different studies. In a cohort of 185 patients, Gautschi et al. demonstrated a significant association between high levels of cfDNA and poor prognosis, but the cut-off was based on measurements in 46 healthy controls, making the transferal into other settings difficult [17]. A study of 46 patients with newly diagnosed, previously untreated NSCLC confirmed these results, while another study of 42 patients did not $[11,18]$. Both studies were limited by a small number of patients. Fournie et al. also found a positive correlation between high levels of cfDNA and poor prognosis, but the study was biased by the mixing of NSCLC and SCLC [19]. In an attempt to address these problems, we performed a consecutive, prospective study including only chemonaive patients with stage III or IV NSCLC. All patients received the same standardized treatment at a single institution and were evaluated by objective criteria, thus constituting a homogenous approach. Furthermore, our study included a reasonable number of patients allowing for analyses of relevant statistical power.

To ensure an independent value of cfDNA we performed multivariate analyses of both PFS and OS. These results further confirmed the prognostic value of baseline
cfDNA, as it remained highly significant in both analyses. In addition to cfDNA, performance status remained an independent prognostic factor, with baseline PS of 2 being significantly related to a poor PFS and OS. This is in consistence with previous knowledge of PS as a welldocumented and clinically used prognostic factor [20]. Also, adenocarcinomas and increased LDH were independent prognostic factors, which is also in accordance with previous knowledge. Disease stage is another known prognostic factor, but showed no correlation with outcome in our patients. This is most likely due to the fact that only patients with stage III and IV disease were included in the study, thereby eliminating the prognostic differences between high and low stages.

The results from the multivariate analysis inspired us to a subgroup analysis combining patients with a high baseline level of cfDNA and a poor PS (PS = 2). This approach, which to our knowledge is the first, attempt to outline a subgroup of patients, who based on these two parameters have a markedly worse prognosis than the rest of the patients. Even though the number of patients in the subgroup was small, it is important to identify those patients who may be better of without treatment.

In conclusion, our results demonstrate a highly significant, independent prognostic value of the baseline cfDNA in patients with advanced, newly diagnosed NSCLC treated with chemotherapy. In a time where treatment is almost mandatory, the level of cfDNA, suggestively in combination with PS, may offer a prognostic grid to help identifying a group of patients who most probably will not benefit from systemic treatment but instead should be offered best supportive care. These intriguing results add new knowledge to the clinical role of cfDNA and call for further investigation and validation.

\section{Acknowledgements}

We thank Yvette Schandorf Sørensen for keeping track of the blood sample collection and Anita Laier Høvenhoff for technical assistance. We thank Karin Larsen for proofreading the final manuscript. There are no funding sources to report.

\section{REFERENCES}

[1] S. S. Hecht, "Tobacco Smoke Carcinogens and Lung Cancer," Journal of the National Cancer Institute, Vol. 91, No. 14, 1999, pp. 1194-1210. doi:10.1093/jnci/91.14.1194

[2] L. A. Gorenstein and J. R. Sonett, "The Surgical Management of Stage I and Stage II Lung Cancer," Surgical Oncology Clinics of North America, Vol. 20, No. 4, 2011, pp. 701-720. doi:10.1016/j.soc.2011.07.009

[3] E. M. Tan, P. H. Schur, R. I. Carr and H. G. Kunkel, "Deoxybonucleic Acid (DNA) and Antibodies to DNA in the 
Serum of Patients with Systemic Lupus Erythematosus," Journal of Clinical Investigation, Vol. 45, No. 11, 1966, pp. 1732-1740. doi:10.1172/JCI105479

[4] S. A. Leon, B. Shapiro, D. M. Sklaroff and M. J. Yaros, "Free DNA in the Serum of Cancer Patients and the Effect of Therapy," Cancer Research, Vol. 37, No. 3, 1977, pp. 646-650.

[5] C. Alix-Panabieres and K. Pantel, "Circulating Tumor Cells: Liquid Biopsy of Cancer," Clinical Chemistry, Vol. 59, No. 1, 2013, pp. 110-118. doi:10.1373/clinchem.2012.194258

[6] M. Stroun, P. Maurice, V. Vasioukhin, J. Lyautey, C. Lederrey, F. Lefort, et al., "The Origin and Mechanism of Circulating DNA," Annals of the New York Academy of Sciences, Vol. 906, 2000, pp. 161-168. doi:10.1111/j.1749-6632.2000.tb06608.x

[7] F. Diehl, M. Li, D. Dressman, Y. He, D. Shen, S. Szabo, et al., "Detection and Quantification of Mutations in the Plasma of Patients with Colorectal Tumors," Proceedings of the National Academy of Sciences of the United States of America, Vol. 102, No. 45, 2005, pp. 16368-16373. doi:10.1073/pnas.0507904102

[8] H. Schwarzenbach, D. S. Hoon and K. Pantel, "Cell-Free Nucleic Acids as Biomarkers in Cancer Patients," Nature Reviews Cancer, Vol. 11, No. 6, 2011, pp. 426-437. doi:10.1038/nrc3066

[9] P. Therasse, S. G. Arbuck, E. A. Eisenhauer, J. Wanders, R. S. Kaplan, L. Rubinstein, et al., "New Guidelines to Evaluate the Response to Treatment in Solid Tumors. European Organization for Research and Treatment of Cancer, National Cancer Institute of the United States, National Cancer Institute of Canada," Journal of the National Cancer Institute, Vol. 92, No. 3, 2000, pp. 205-216. doi:10.1093/jnci/92.3.205

[10] K. L. Spindler, N. Pallisgaard, I. Vogelius and A. Jakobsen, "Quantitative Cell-Free DNA, KRAS, and BRAF Mutations in Plasma from Patients with Metastatic Colorectal Cancer during Treatment with Cetuximab and Irinotecan," Clinical Cancer Research, Vol. 18, No. 4, 2012, pp. 1177-1185. doi:10.1158/1078-0432.CCR-11-0564

[11] S. Kumar, R. Guleria, V. Singh, A. C. Bharti, A. Mohan and B.-C. Das, "Plasma DNA Level in Predicting Therapeutic Efficacy in Advanced Nonsmall Cell Lung Cancer," European Respiratory Journal, Vol. 36, No. 4, 2010, pp. 885-892. doi:10.1183/09031936.00187909

[12] G. Pelosi, E. Schianchi, P. Dell'orto, G. Veronesi, L. Spaggiari, F. Pasini, et al., "Detecting Cell-Free Circulating hTERT mRNA in the Plasma May Identify a Subset of Nonsmall Cell Lung Cancer Patients," Virchows Arch, Vol. 448, No. 1, 2006, pp. 7-15. doi: $10.1007 / \mathrm{s} 00428-005-0087-\mathrm{z}$

[13] C. Camps, R. Sirera, R. M. Bremnes, V. Rodenas, A. Blasco, M. J. Safont, et al., "Quantification in the Serum of the Catalytic Fraction of Reverse Telomerase: A Useful Prognostic Factor in Advanced Non-Small Cell Lung Cancer," Anticancer Research, Vol. 26, No. 6C, 2006, pp. 4905-4909.

[14] R. Sirera, R. M. Bremnes, A. Cabrera, E. Jantus-Lewintre, E. Sanmartin, A. Blasco, et al., "Circulating DNA Is a Useful Prognostic Factor in Patients with Advanced NonSmall Cell Lung Cancer," Journal of Thoracic Oncology, Vol. 6, No. 2, 2011, pp. 286-290. doi:10.1097/JTO.0b013e31820189a5

[15] R. M. Van den Berg, H. Brokx, A. Vesin, J. K. Field, C. Brambilla, C. J. Meijer, et al., "Prognostic Value of hTERT mRNA Expression in Surgical Samples of Lung Cancer Patients: The European Early Lung Cancer Project," International Journal of Oncology, Vol. 37, No. 2, 2010, pp. 455-461.

[16] A. K. Pathak, M. Bhutani, S. Kumar, A. Mohan and R. Guleria, "Circulating Cell-Free DNA in Plasma/Serum of Lung Cancer Patients as a Potential Screening and Prognostic Tool," Clinical Chemistry, Vol. 52, No. 10, 2006, pp. 1833-1842.

[17] O. Gautschi, C. Bigosch, B. Huegli, M. Jermann, A. Marx, E. Chasse, et al., "Circulating Deoxyribonucleic Acid as a Prognostic Marker in Non-Small-Cell Lung Cancer Patients Undergoing Chemotherapy," Journal of Clinical Oncology, Vol. 22, No. 20, 2004, pp. 4157-4164. doi:10.1200/JCO.2004.11.123

[18] M. A. van der Drift, B. E. Hol, C H. Klaassen, C. F. Prinsen, Y. A. van Aarssen, R. Donders, et al., "Circulating DNA Is a Non-Invasive Prognostic Factor for Survival in Non-Small Cell Lung Cancer," Lung Cancer, Vol. 68, No. 2, 2010, pp. 283-287. doi:10.1016/j.lungcan.2009.06.021

[19] G. J. Fournie, J. P. Courtin, F. Laval, J. J. Chale, J. P. Pourrat, M. C. Pujazon, et al., "Plasma DNA as a Marker of Cancerous Cell Death. Investigations in Patients Suffering from Lung Cancer and in Nude Mice Bearing Human Tumours," Cancer Letters, Vol. 91, No. 2, 1995, pp. 221-227. doi:10.1016/0304-3835(95)03742-F

[20] M. D. Brundage, D. Davies and W. J. Mackillop, "Prognostic Factors in Non-Small Cell Lung Cancer: A Decade of Progress," Chest, Vol. 122, No. 3, 2002, pp. 1037-1057. doi:10.1378/chest.122.3.1037 